\title{
Emergence of Community-Acquired Methicillin-Resistant Staphylococcus aureus USA 300 Clone as the Predominant Cause of Skin and Soft-Tissue Infections
}

Mark D. King, MD, MS; Bianca J. Humphrey, BS; Yun F. Wang, PhD; Ekaterina V. Kourbatova, MD, MPH; Susan M. Ray, MD; and Henry M. Blumberg, MD

Background: Studies have shown that community-acquired methicillin-resistant Staphylococcus aureus (MRSA) causes S. aureus skin and soft-tissue infection in selected populations.

Objective: To determine the proportion of infections caused by community-acquired MRSA, the clinical characteristics associated with community-acquired MRSA, and the molecular epidemiology of community-acquired MRSA among persons with communityonset $S$. aureus skin and soft-tissue infection.

Design: Active, prospective laboratory surveillance to identify $S$. aureus recovered from skin and soft-tissue sources.

Setting: 1000-bed urban hospital and its affiliated outpatient clinics in Atlanta, Georgia.

Patients: 384 persons with microbiologically confirmed communityonset $S$. aureus skin and soft-tissue infection.

Measurements: Proportion of infections caused by and clinical factors associated with community-acquired MRSA among persons with community-onset $S$. aureus skin and soft-tissue infection. Pulsed-field gel electrophoresis and antimicrobial susceptibility patterns were used to epidemiologically classify community-onset $S$. aureus infections. Community-acquired MRSA was defined by MRSA isolates that either demonstrated a USA 300 or USA 400 pulsed-field type or had a susceptibility pattern showing resistance only to $\beta$-lactams and erythromycin (for isolates not available for pulsed-field gel electrophoresis).

Results: Community-onset skin and soft-tissue infection due to $S$. aureus was identified in 389 episodes, with MRSA accounting for
$72 \%$ (279 of 389 episodes). Among all S. aureus isolates, 63\% (244 of 389 isolates) were community-acquired MRSA. Among MRSA isolates, $87 \%$ (244 of 279 isolates) were community-acquired MRSA. When analysis was restricted only to MRSA isolates that were available for pulsed-field gel electrophoresis, 91\% (159 of 175 isolates) had a pulsed-field type consistent with communityacquired MRSA; of these, 99\% (157 of 159 isolates) were the MRSA USA 300 clone. Factors independently associated with community-acquired MRSA infection were black race (prevalence ratio, $1.53[95 \% \mathrm{Cl}, 1.16$ to 2.02$]$ ), female sex (prevalence ratio, 1.16 $[\mathrm{Cl}, 1.02$ to 1.32$])$, and hospitalization within the previous 12 months (prevalence ratio, $0.80[\mathrm{Cl}, 0.66$ to 0.97$]$ ). Inadequate initial antibiotic therapy was statistically significantly more common among those with community-acquired MRSA (65\%) than among those with methicillin-susceptible $S$. aureus skin and soft-tissue infection (1\%).

Limitations: Some MRSA isolates were not available for molecular typing.

Conclusions: The community-acquired MRSA USA 300 clone was the predominant cause of community-onset S. aureus skin and soft-tissue infection. Empirical use of agents active against community-acquired MRSA is warranted for patients presenting with serious skin and soft-tissue infections.
Ann Intern Med. 2006;144:309-317.

For author affiliations, see end of text.

www.annals.org

field type, while outbreaks associated with severe and fatal disease in children, as well as skin and soft-tissue infections in Native American populations, have been associated with the USA 400 pulsed-field type (6).

The community-acquired MRSA clones have frequently been associated with the Panton-Valentine leuko-

See also:

Print

Editors' Notes . . . . . . . . . . . . . . . . . . . . . . 310

Editorial comment. . . . . . . . . . . . . . . . . . . 368

Related article. . . . . . . . . . . . . . . . . 318

Summary for Patients. . . . . . . . . . . . . . . . I-11

Web-Only

CME quiz

Conversion of figures and tables into slides athletes have been associated with the USA 300 pulsed- 


\section{Context}

In community outbreaks of methicillin-resistant Staphylococcus aureus (MRSA), 2 clones predominate in the United States: USA 300 and USA 400. Little is known about these infections in the nonoutbreak setting.

\section{Contribution}

This study evaluated nonoutbreak community-acquired $S$. aureus skin and soft-tissue infections in patients in a large urban setting. Almost three quarters of the soft-tissue infections were caused by MRSA, and these were predominantly the USA 300 type. No MRSA USA 300 isolate was resistant to trimethoprim-sulfamethoxazole or vancomycin, while most were resistant to erythromycin in addition to $\beta$-lactams.

\section{Implications}

Antibiotic choice for serious nonoutbreak community-acquired skin and soft-tissue infection should consider high rates of MRSA in some communities.

- The Editors

cidin virulence factor and the presence of staphylococcal chromosome cassette mec (SCCmec) type IV allele (1, 6-9). In contrast, hospital-acquired or health care-associated MRSA strains usually lack genes for Panton-Valentine leukocidin and are associated with other SCCmec alleles (for example, SCCmec type II) $(1,6,7)$. In addition to Panton-Valentine leukocidin and SCCmec type IV, the community-acquired MRSA USA 300 and USA 400 genotypes have usually demonstrated resistance to $\beta$-lactams and erythromycin while retaining susceptibility to clindamycin, trimethoprim-sulfamethoxazole, and fluoroquinolones, whereas health care-associated genotypes are often multidrug-resistant $(1,6)$.

Although increasingly reported as a cause of outbreaks of skin and soft-tissue infection, the proportion of $S$. aureus skin and soft-tissue infections caused by community-acquired MRSA in nonoutbreak settings remains poorly defined. We sought to determine the proportion of infections caused by community-acquired MRSA, the clinical characteristics associated with community-acquired MRSA, and the molecular epidemiology of community-acquired MRSA among persons with community-onset $S$. aureus skin and soft-tissue infection who were receiving care at a large hospital and its affiliated clinics in urban Atlanta, Georgia.

\section{Methods}

\section{Laboratory Surveillance for Community-Onset $S$. aureus Skin and Soft-Tissue Infection}

We conducted active, prospective laboratory surveillance from 1 August 2003 to 15 November 2003 to identify $S$. aureus isolates of patients with skin and soft-tissue infections at the Grady Health System. The Grady Health System includes Grady Memorial Hospital, a 1000-bed public inner-city hospital, and its affiliated outpatient clinics. We limited surveillance to $S$. aureus isolates that were obtained from patients with a community-onset skin and soft-tissue infection (isolates designated either as an exudate or a body fluid culture). We defined community-onset $S$. aureus by positive test results from cultures that were obtained within 72 hours of admission or at an outpatient visit. An investigator classified community-onset $S$. aureus before we performed molecular typing studies or before we reviewed antimicrobial susceptibility results other than methicillin susceptibility. We identified $S$. aureus and tested antimicrobial susceptibility for all isolates in the Grady Memorial Hospital Clinical Microbiology Laboratory in accordance with the National Committee for Clinical Laboratory Standards (10).

\section{Study Sample and Data Collection}

The Emory University Institutional Review Board and the Grady Research Oversight Committee approved the study. Grady Memorial Hospital provides medical care for a primarily medically indigent inner-city population, approximately $80 \%$ of whom are African American, residing within inner-city Atlanta (Fulton County and DeKalb County). The hospital serves as a teaching hospital for both Emory University School of Medicine and Morehouse School of Medicine. The referral pattern for the hospital and its affiliated clinics is primarily patient self-referral on the basis of place of residence (that is, residence within DeKalb or Fulton Counties) or indigent status, with some patients referred for tertiary academic medical care. We retrospectively reviewed computerized medical and laboratory records to document patient demographic characteristics, HIV status, presence of end-stage renal disease, hospitalization within the previous 12 months, and any history of MRSA infection or colonization. The reviewer completed the abstraction process blinded to the molecular typing results of MRSA isolates. We documented the need for hospitalization for managing the $S$. aureus skin and softtissue infection. We obtained $S$. aureus antimicrobial susceptibility profiles from the hospital's microbiology laboratory, and we categorized infections as due to either methicillin-susceptible $S$. aureus (MSSA) or MRSA. We reviewed computerized pharmacy records to document the antimicrobial agent therapy that was used to treat the patient's skin and soft-tissue infection. We considered antimicrobial agent therapy to be adequate if the prescribed antimicrobial agent had in vitro activity against the isolated $S$. aureus strain.

\section{Molecular Typing and Genetic Analyses}

We performed pulsed-field gel electrophoresis (PFGE) using SmaI as a restriction endonuclease on all available MRSA isolates, as described by Bannerman and colleagues (11). We digitized gels and saved them as an image for analysis with BioNumerics software (Applied Maths, Sint- 
Martens-Latem, Belgium). We performed cluster analysis by using the unweighted pair-group method based on Dice coefficients. We defined pulsed-field type clusters by using a similarity coefficient of at least $80 \%$ and the criteria of Tenover and colleagues (12). By using the nomenclature outlined by McDougal and colleagues (6), we categorized MRSA isolates into 1 of 8 lineages: USA 100 through USA 800.

For some isolates, we determined SCCmec type by using polymerase chain reaction (PCR)-typing of the mec gene complex as described by Okuma and colleagues (7). Similarly, we assessed the presence of Panton-Valentine leukocidin genes in selected MRSA isolates by using PCR as previously described by Lina and colleagues (13).

\section{Classification of Community-Onset S. aureus Skin and Soft-Tissue Infections}

We classified community-onset $S$. aureus skin and softtissue infections into 3 groups on the basis of either molecular typing results (pulsed-field type) of MRSA isolates or antimicrobial susceptibility pattern in cases in which the MRSA isolate was not available for PFGE. The 3 groups were 1) the community-acquired MRSA USA 300/USA 400 group, 2) the "other MRSA" group, and 3) the MSSA group. The community-acquired MRSA USA 300/USA 400 group was defined by MRSA skin and soft-tissue infections caused by a MRSA isolate that either demonstrated a USA 300 or USA 400 pulsed-field type (6) or had an antimicrobial susceptibility profile demonstrating resistance only to $\beta$-lactams and erythromycin while retaining susceptibility to clindamycin, levofloxacin, trimethoprimsulfamethoxazole, and vancomycin. The "other MRSA" group was defined by MRSA skin and soft-tissue infections caused by a MRSA isolate that either demonstrated a pulsed-field type other than USA 300 or USA 400 (for example, USA 100, USA 500, or USA 800 [which have all been described as health care-associated pulsed-field types]) or had an antimicrobial susceptibility profile demonstrating resistance to $\beta$-lactams, erythromycin, and at

Figure 1. Clinical and molecular epidemiology of Staphylococcus aureus skin and soft-tissue infection.

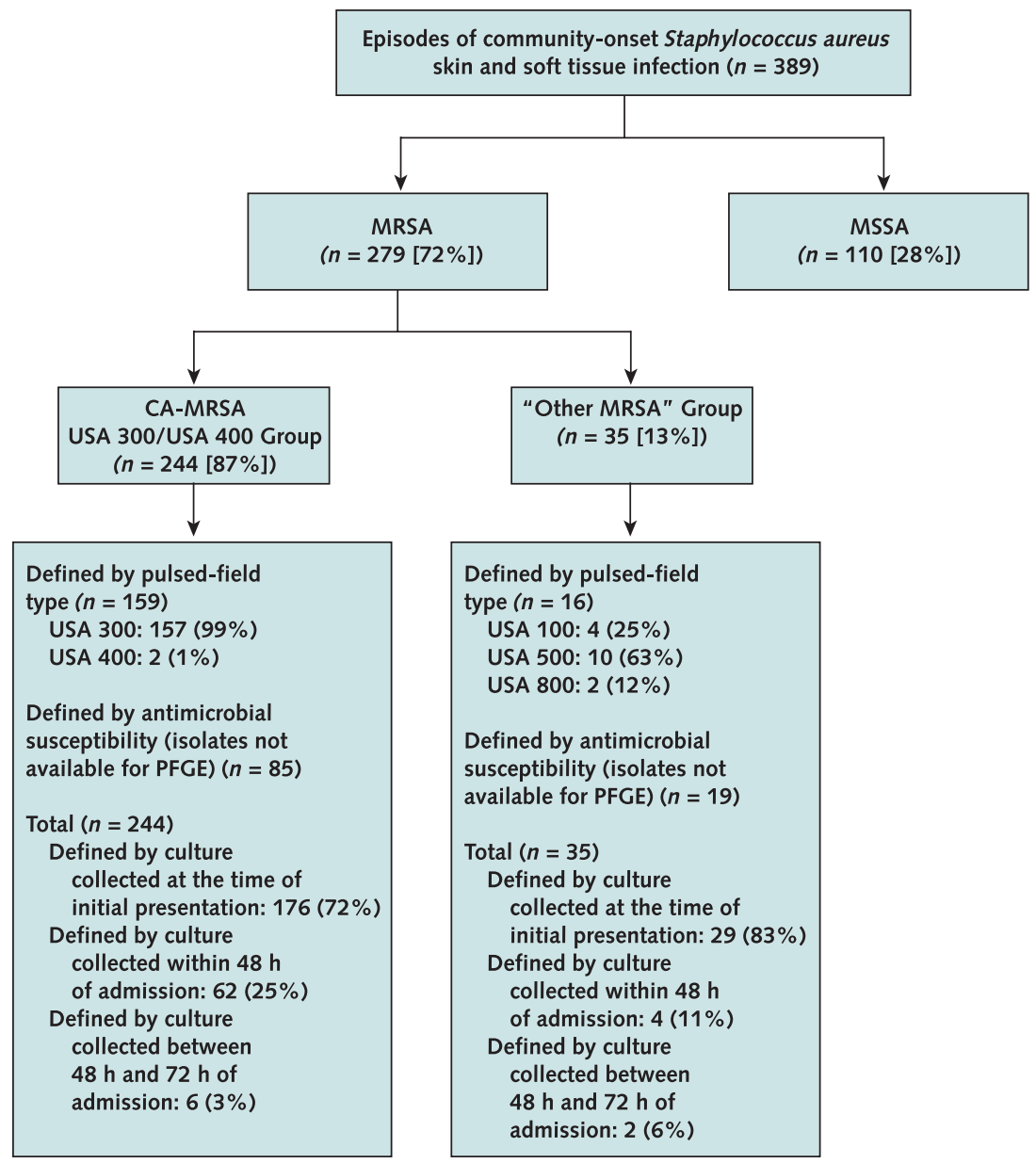

CA-MRSA = community-acquired methicillin-resistant Staphylococcus aureus; MRSA = methicillin-resistant Staphylococcus aureus; MSSA = methicillinsusceptible Staphylococcus aureus; PFGE = pulsed-field gel electrophoresis. 
Figure 2. Dendrogram of representative pulsed-field types from methicillin-resistant Staphylococcus aureus isolates causing skin and soft-tissue infections.

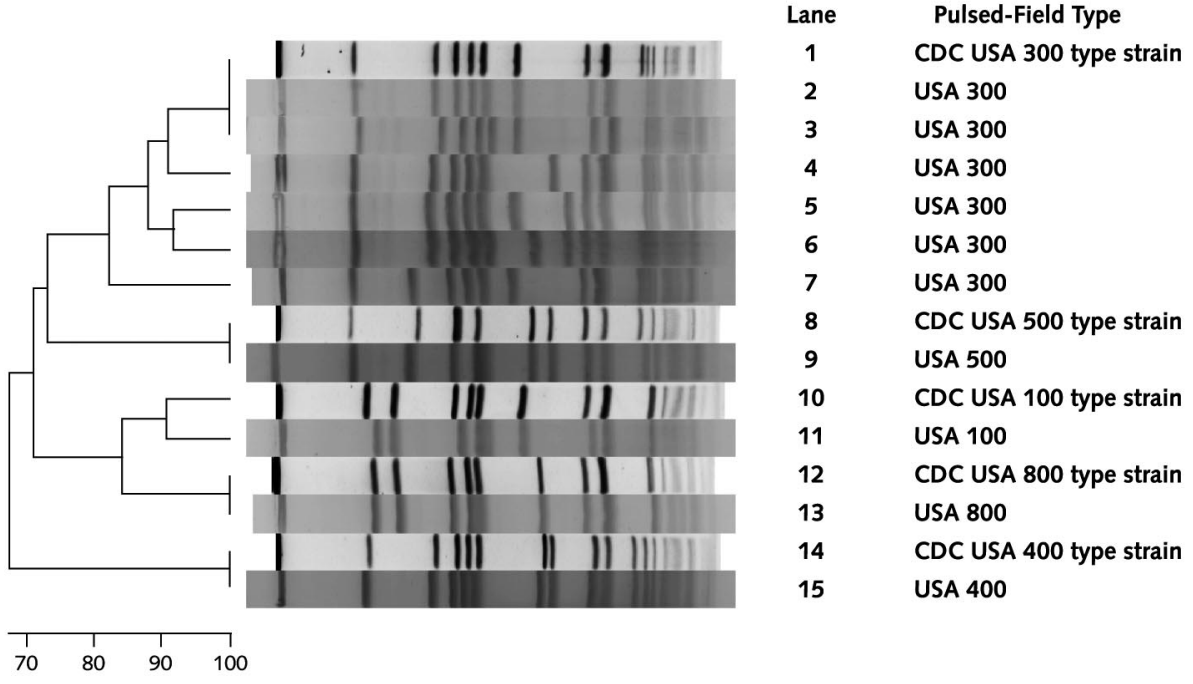

The figure shows infections among patients seen at Grady Memorial Hospital (lanes 2 to 7, 9, 11, 13, and 15) and representative methicillin-resistant Staphylococcus aureus standard-type strains previously published by the Centers for Disease Control and Prevention $(C D C)($ lanes $1,8,10,12$, and 14$)$ (6). CDC data from McDougal et al. (6).

least 1 additional antibiotic. We defined the MSSA group by skin and soft-tissue infections caused by MSSA.

\section{Statistical Analysis}

We performed data management and statistical analyses by using Microsoft Excel 2000 software (Microsoft Corp., Redmond, Washington) and SAS software, version 8.2 (SAS Institute, Cary, North Carolina). We assessed risk factors for community-acquired MRSA skin and soft-tissue infection in 2 separate analyses. Our first analysis compared the community-acquired MRSA USA 300/USA 400 group with the MSSA group, and our second analysis compared the community-acquired MRSA USA 300/USA 400 group with the other MRSA group. In addition, we repeated each analysis using data that were limited to those skin and soft-tissue infections in which a MRSA isolate was available for PFGE to account for any potential misclassification bias introduced when skin and soft-tissue infections were classified according to antimicrobial susceptibility profile.

We initially identified potential risk factors for community-acquired MRSA skin and soft-tissue infection by univariate analysis. We calculated prevalence ratios and the corresponding 95\% CIs. Multivariable log-binomial regression models included variables that were statistically significantly associated with community-acquired MRSA skin and soft-tissue infection in univariate analysis, as well as potential confounders and effect modifiers. We tested interaction among main effect covariates by using 2-way interaction terms, and we assessed confounding. We chose the variables included in the final model a priori on the basis of the biological plausibility of their association with the outcome of interest, as well as on the basis of statistical and epidemiologic criteria. We defined a $P$ value of 0.05 or less as being statistically significant.

\section{Role of the Funding Sources}

The Emory Medical Care Foundation, Emory Mentored Clinical Research Scholars Program, National Institutes of Health, National Center for Research Resources, and National Institute of Allergy and Infectious Diseases provided funding for the study. The funding sources had no role in the study design, data collection, data analysis, and data interpretation or in the writing of the report.

\section{RESULTS \\ Clinical and Molecular Epidemiology of Community-Onset S. aureus Skin and Soft-Tissue Infection}

We identified microbiologically confirmed community-onset $S$. aureus skin and soft-tissue infection in $389 \mathrm{ep}-$ isodes of skin and soft-tissue infection among 384 persons. Of these episodes, 279 (72\%) episodes were caused by MRSA and 110 (28\%) episodes were caused by MSSA (Figure 1). Among the 389 community-onset S. aureus skin and soft-tissue infections, $244(63 \%)$ were classified within the community-acquired MRSA USA 300/USA 400 group (159 infections by pulsed-field type and 85 infections by antimicrobial susceptibility profile [for isolates not available for PFGE]). Among the 279 communityonset MRSA skin and soft-tissue infections, 244 (87\%) 
infections were classified within the community-acquired MRSA USA 300/USA 400 group and 35 (13\%) infections were classified within the other MRSA group (Figure 1).

When we considered only isolates in which PFGE was performed, 159 of 175 (91\%) MRSA isolates had a pulsedfield type consistent with community-acquired MRSA USA 300 or USA 400 pulsed-field type and 16 (9\%) MRSA isolates had non-community-associated or health care-associated pulsed-field types (Figures 1 and 2). The predominant community-acquired MRSA pulsed-field type was USA 300, which accounted for 157 of 159 (99\%) community-acquired MRSA isolates studied (Figure 2, lanes 2 to 7). The other 2 community-acquired MRSA isolates had a USA 400 pulsed-field type (Figure 2, lane 15). Among the 157 isolates with a USA 300 pulsed-field type, $121(77 \%)$ isolates were identical to a CDC USA 300 type strain (Figure 2, lanes 2 and 3) and $36(23 \%)$ isolates were consistent with USA 300 variant strains (Figure 2, lanes 4 to 7) on the basis of coefficients of similarity of $80 \%$ or higher and the criteria of Tenover and colleagues (12). Figure 2 (lanes 9, 11, and 13) also shows the pulsedfield types that are representative of non-community-acquired MRSA strains (USA 500, USA 100, and USA 800) obtained from MRSA isolates of patients with skin and softtissue infections at Grady Memorial Hospital. Two of 2 (100\%) community-acquired MRSA USA 300 isolates tested had Panton-Valentine leukocidin and SCCmec type IV.

\section{Antimicrobial Susceptibility Patterns of MRSA Isolates according to Pulsed-Field Type}

Most USA 300 isolates demonstrated resistance only to $\beta$-lactams and erythromycin (136 of 157 [87\%] isolates), with fewer demonstrating resistance to levofloxacin (15 of 157 [10\%] isolates), clindamycin (4 of 157 [3\%] isolates), rifampin (1 of 157 [1\%] isolates), or gentamicin (1 of 157 [1\%] isolates) (Table 1). No USA 300 isolate was resistant to trimethoprim-sulfamethoxazole or vancomycin, and 2 of $157(1 \%)$ isolates demonstrated resistance only to $\beta$-lactams. In contrast, MRSA isolates with noncommunity-associated or "health care-associated" pulsedfield types usually were resistant to other antibiotics in addition to $\beta$-lactams and erythromycin (14 of 16 [88\%] isolates) (Table 1).

\section{Characteristics of Patients with S. aureus Skin and Soft-Tissue Infection}

Table 2 shows the characteristics of persons in the community-acquired MRSA USA 300/USA 400 group, the other MRSA group, and the MSSA group. When compared with the MSSA group in univariate analysis, persons in the community-acquired MRSA USA 300/USA 400 group were more likely to be black and female. Persons in the community-acquired MRSA USA 300/USA 400 group were less likely to have been hospitalized within the previous 12 months than those in the MSSA group (Table 2). Age, HIV status, or the frequency of previous infection or colonization with MRSA did not differ between the com-

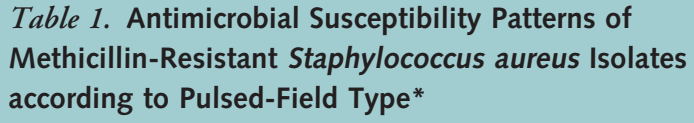

Resistance Pattern

Community-Acquired MRSA USA 300 Group, n (\%)

$\beta$-Lactam and erythromycin

$\beta$-Lactam, erythromycin, and $\geq 1$ additional antibiotic

$\beta$-Lactam only 136 (87) $19(12)$ 2 (1)

* Pulsed-field types are defined in accordance with nomenclature outlined by McDougal et al. (6). MRSA = methicillin-resistant Staphylococcus aureus.

† The other MRSA group represents MRSA isolates with pulsed-field types other than USA 300 or USA 400. These included USA 100, USA 500, and USA 800 and are representative of health care-associated genotypes.

munity-acquired MRSA USA 300/USA 400 group and the MSSA group. When compared with the other MRSA group, persons in the community-acquired MRSA USA 300/USA 400 group were more likely to be younger than 50 years of age, to be black, and to be HIV-seronegative. As observed in the MSSA comparison, persons in the community-acquired MRSA USA 300/USA 400 group were also less likely than those in the other MRSA group to have been hospitalized in the previous 12 months (Table 2). Sex or the frequency of previous infection or colonization with MRSA did not statistically significantly differ between the MRSA groups. Inadequate empirical and definitive antibiotic therapy (that is, use of agents without in vitro activity against the $S$. aureus isolate recovered from the patient) were much more common among patients with community-acquired MRSA (65\% and 43\%, respectively) than among those in the MSSA group $(1 \%$ and $1 \%$, respectively).

\section{Multivariate Analyses}

Black race (prevalence ratio, 1.53 [95\% CI, 1.16 to 2.02]), female sex (prevalence ratio, 1.16 [CI, 1.02 to 1.32]), and hospitalization within the previous 12 months (prevalence ratio, 0.80 [CI, 0.66 to 0.97$]$ ) were independently associated with the community-acquired MRSA USA 300/USA 400 group compared with the MSSA group in multivariate analysis (Table 3 ). When we repeated the analysis by using data that were limited to those with skin and soft-tissue infections in which a MRSA isolate was available for PFGE, black race (prevalence ratio, 1.87 [CI, 1.25 to 2.81 ]), female sex (prevalence ratio, 1.25 [CI, 1.04 to 1.50]), and hospitalization within the previous 12 months (prevalence ratio, 0.71 [CI, 0.53 to 0.95$]$ ) remained independently associated with the community-acquired MRSA USA 300/USA 400 group (Table 3). The few cases in the other MRSA group limited construction of multivariate models comparing the community-acquired MRSA USA 300/USA 400 group with the other MRSA group; therefore, we present results of only the univariate analysis comparing these groups (Table 2 ). 
ARTICLE $\mid$ Community-Acquired MRSA Skin and Soft-Tissue Infections

Table 2. Characteristics of Patients with Staphylococcus aureus Skin and Soft-Tissue Infection and Univariate Risk Factors for Community-Acquired Methicillin-Resistant S. aureus USA 300/USA 400 Skin and Soft-Tissue Infection*

\begin{tabular}{|c|c|c|c|c|c|}
\hline Characteristic & $\begin{array}{l}\text { Community-Acquired } \\
\text { MRSA USA } 300 / \\
\text { USA } 400 \text { Group } \\
(n=244)\end{array}$ & $\begin{array}{l}\text { MSSA Group } \\
(n=110)\end{array}$ & $\begin{array}{l}\text { Prevalence Ratio } \\
(95 \% \mathrm{Cl})+\end{array}$ & $\begin{array}{l}\text { Other MRSA } \\
\text { Group } \\
(n=35)\end{array}$ & $\begin{array}{l}\text { Prevalence Ratio } \\
(95 \% \mathrm{Cl}) \ddagger\end{array}$ \\
\hline \multicolumn{6}{|l|}{ Sex, $n(\%)$} \\
\hline Female & $123(50)$ & $40(36)$ & $1.19(1.04-1.37)$ & $15(43)$ & $1.04(0.95-1.14)$ \\
\hline Male & $121(50)$ & $70(64)$ & & $20(57)$ & \\
\hline \multicolumn{6}{|l|}{ Race, $n(\%)$} \\
\hline Black & $216(89)$ & $79(72)$ & $1.54(1.17-2.04) \S$ & $25(71)$ & $1.22(1.00-1.48) \S$ \\
\hline White & $20(8)$ & $13(12)$ & & $9(26)$ & \\
\hline Other & $8(3)$ & $18(16)$ & & $1(3)$ & \\
\hline Median age (range), $y \|$ & $34(0-70)$ & $36(0-94)$ & - & $45(0-84)$ & - \\
\hline Age group, $n(\%)$ & & & $1.16(0.93-1.45) 9$ & & $1.25(1.04-1.50) 9$ \\
\hline$\leq 2 y$ & $22(9)$ & $10(9)$ & & $3(9)$ & \\
\hline $3-5 y$ & $5(2)$ & $7(6)$ & & $0(0)$ & \\
\hline $6-17 y$ & $36(15)$ & $9(8)$ & & $0(0)$ & \\
\hline $18-34 y$ & $63(26)$ & $27(25)$ & & $10(28)$ & \\
\hline $35-49 y$ & $84(34)$ & $35(32)$ & & $9(26)$ & \\
\hline $50-64 y$ & $31(13)$ & $13(12)$ & & $10(28)$ & \\
\hline$\geq 65 y$ & $3(1)$ & $9(8)$ & & $3(9)$ & \\
\hline HIV negative, $n(\%)^{* *}$ & $203(83)$ & $88(80)$ & $1.07(0.88-1.30)$ & $21(60)$ & $1.25(1.04-1.50)$ \\
\hline ESRD, $n(\%)$ & $0(0)$ & $2(2)$ & - & $0(0)$ & - \\
\hline MRSA bacteremia, $n(\%)$ & $5(2)$ & $4(4)$ & $0.80(0.45-1.44)$ & $1(3)$ & $0.95(0.66-1.37)$ \\
\hline $\begin{array}{l}\text { Hospitalized in previous } 12 \mathrm{mo} \\
n(\%)\end{array}$ & 45 (18) & $32(29)$ & $0.81(0.66-1.00)$ & $20(57)$ & $0.74(0.63-0.88)$ \\
\hline $\begin{array}{l}\text { History of MRSA infection or } \\
\text { colonization, } n(\%)\end{array}$ & $23(9)$ & $7(6)$ & $1.12(0.91-1.39)$ & $6(17)$ & $0.95(0.74-1.09)$ \\
\hline Required hospitalization, $n(\%)$ & $99(41)$ & $49(45)$ & $0.95(0.82-1.10)$ & $19(54)$ & $0.93(0.85-1.02)$ \\
\hline \multicolumn{6}{|l|}{ Antibiotic therapy, $n(\%)+\dagger$} \\
\hline Initial active & $62(35)$ & $68(99)$ & - & $11(50)$ & $0.93(0.83-1.04)$ \\
\hline Ultimate active & $100(57)$ & $68(99)$ & - & $17(77)$ & $0.92(0.83-1.00)$ \\
\hline
\end{tabular}

* ESRD = end-stage renal disease; MRSA = methicillin-resistant Staphylococcus aureus; MSSA = methicillin-susceptible Staphylococcus aureus.

† Prevalence ratio for comparison of community-acquired MRSA USA 300/USA 400 group vs. MSSA group.

₹ Prevalence ratio for comparison of community-acquired MRSA USA 300/USA 400 group vs. other MRSA group.

$\S$ Prevalence ratio represents comparison of black race with white race and other race combined.

$\| P<0.001$ for median age comparison of community-acquired MRSA USA 300/USA 400 vs. other MRSA.

ๆ Prevalence ratio for comparison of age $<50 \mathrm{y}$ to age $\geq 50 \mathrm{y}$.

* * HIV negative encompasses persons with a known HIV-negative status or an unknown HIV status. HIV status known for 92 of 244 patients (38\%) in the communityacquired MRSA group, 37 of 110 patients (34\%) in the MSSA group, and 17 of 35 patients (49\%) in the other MRSA group.

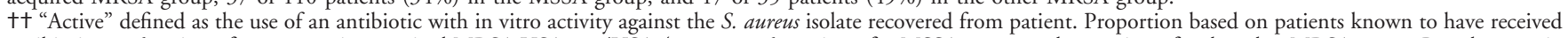
antibiotics: 176 patients for community-acquired MRSA USA 300/USA 400 group; 69 patients for MSSA group; and 22 patients for the other MRSA group. Prevalence ratio

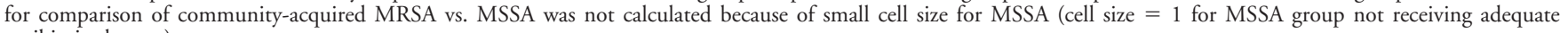
antibiotic therapy).

\section{DISCUSSION}

Over 3.5 months, $72 \%$ of all community-onset $S$. aureus skin and soft-tissue infections at a large public hospital and its affiliated clinics in urban Atlanta were caused by MRSA. We have shown that community-acquired MRSA, primarily the USA 300 clone, was the predominant cause of community-onset $S$. aureus skin and soft-tissue infection at Grady Memorial Hospital. Community-acquired MRSA accounted for $63 \%$ of all S. aureus skin and soft-tissue infections and $87 \%$ of MRSA skin and soft-tissue infections. However, most clinicians did not recognize that community-acquired MRSA emerged as the leading cause of community-onset skin and soft-tissue infection, as evidenced by the high proportion of community-acquired MRSA infections treated with antimicrobial agents lacking activity against MRSA (primarily $\beta$-lactams).

The proportion of skin and soft-tissue infections caused by community-acquired MRSA at Grady Memorial
Hospital in 2003 was much higher than that reported in a population-based study conducted in Minnesota in 2000, in which $22 \%$ of MRSA isolates from skin and soft-tissue infection were community-acquired MRSA (1). Although previous reports of community-acquired MRSA skin and soft-tissue infection have primarily been limited to outbreaks in selected populations, such as injection drug users, homosexual men, those incarcerated in correctional facilities, Native Americans, and children $(2-5,9)$, our data indicate that community-acquired MRSA has now become a widespread and endemic cause of $S$. aureus skin and softtissue infection in our community. Data from San Francisco also suggest that community-acquired MRSA strains are increasingly prevalent, with $69 \%$ of MRSA strains harboring Panton-Valentine leukocidin genes recovered from skin and soft-tissue sources from patients who attended a clinic that specializes in skin and soft-tissue infections (8). In this specialized clinic, with many injection drug users, 
$76 \%$ of the $S$. aureus isolates from skin and soft-tissue cultures were MRSA, a prevalence similar to our observations in urban Atlanta (14).

In areas with a high prevalence of community-acquired MRSA, empirical use of agents that are active against community-acquired MRSA (for example, vancomycin) is now warranted for patients who present with serious skin and soft-tissue infections. The emergence of community-acquired MRSA as an endemic cause of community-onset skin and soft-tissue infection has led to modifications of our institutional guidelines for treating skin and soft-tissue infections. These guidelines now recommend vancomycin as a first-line agent for patients who are hospitalized with a serious skin and soft-tissue infection. Previously (when MSSA was the predominant cause of community-onset staphylococcal skin and soft-tissue infections), vancomycin was not a recommended antimicrobial agent for treating community-onset skin and soft-tissue infection because of concerns about the effect of inappropriate vancomycin use on glycopeptide resistance in grampositive organisms, including $S$. aureus $(15,16)$. Given the potential ramifications of increased vancomycin use, clinicians should obtain specimens for culture in this setting so that infections caused by MSSA or streptococci can be treated with a $\beta$-lactam rather than vancomycin or other non- $\beta$-lactam agents. In areas with a high prevalence of community-acquired MRSA, clinicians should use non- $\beta$ lactam agents for localized skin and soft-tissue infections, particularly for those associated with abscess formation, which are amenable to outpatient therapy and require primary or adjunctive antimicrobial agent therapy. Some studies have suggested use of clindamycin, trimethoprimsulfamethoxazole, or linezolid for the outpatient treatment of skin and soft-tissue infections due to MRSA (17). However, the efficacy of nonglycopeptide antimicrobial agents in treating MRSA skin and soft-tissue infections remains incompletely defined, and clinical trials are needed to better define their role.

Concerns about inducible clindamycin resistance via erm genes among community-acquired MRSA isolates that are resistant to macrolides but demonstrate in vitro susceptibility to clindamycin have made many clinicians reluctant to treat community-acquired MRSA infections with clindamycin. Inducible resistance mediated by erm genes occurs by modification of ribosomal drug-binding sites, resulting in resistance to macrolides, lincosamides, and group B streptogramins, and has been called the MSLBi phenotype (18). Although data are limited, clinical failures of clindamycin treatment for community-acquired MRSA isolates with a MSLBi phenotype have been reported, which suggests that clindamycin probably should be avoided for treating infections caused by isolates with an MSLBi phenotype $(18,19)$. In contrast, efflux pump-mediated macrolide resistance in $S$. aureus (msr[A]) does not lead to inducible clindamycin resistance, and infections caused by such isolates can be treated with clindamycin

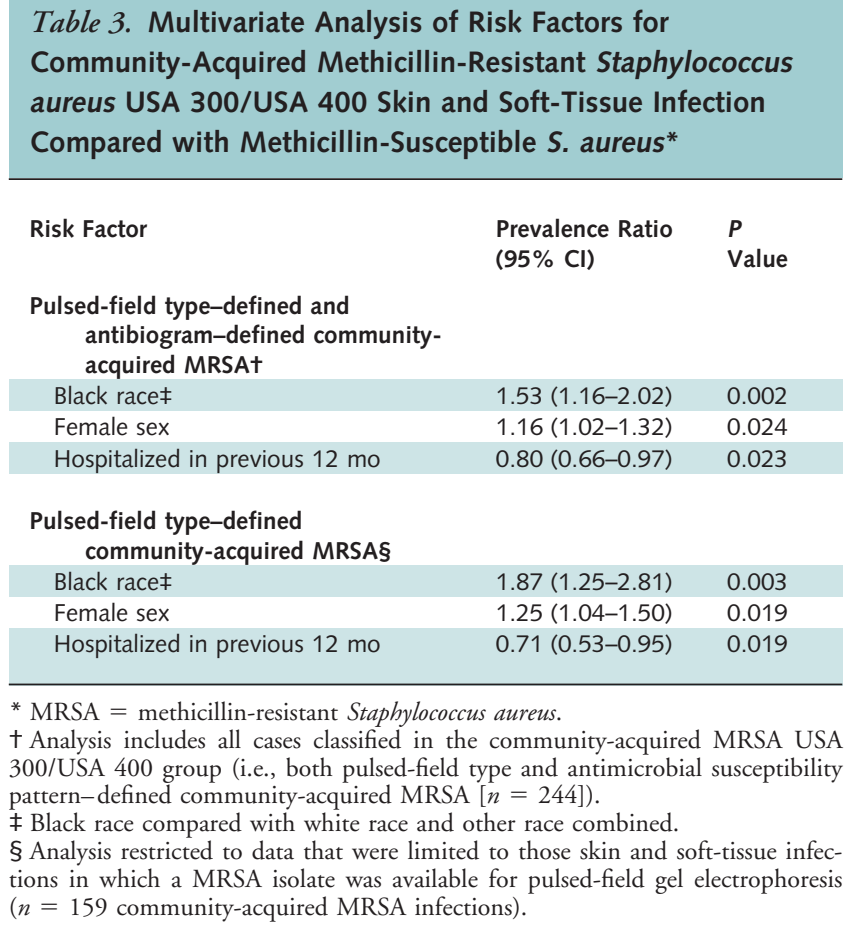

Risk Factor

Prevalence Ratio $P$ $(95 \% \mathrm{Cl})$

Value

Pulsed-field type-defined and antibiogram-defined communityacquired MRSAT

Black raceł

Female sex

Hospitalized in previous $12 \mathrm{mo}$

$1.53(1.16-2.02)$

$1.16(1.02-1.32)$

$0.80(0.66-0.97)$

0.023

Pulsed-field type-defined

community-acquired MRSA§

Black race $\neq$

$1.87(1.25-2.81)$

$1.25(1.04-1.50)$

Female sex

$0.71(0.53-0.95)$

0.003

0.019

Hospitalized in previous $12 \mathrm{mo}$

0.019

* MRSA = methicillin-resistant Staphylococcus aureus.

† Analysis includes all cases classified in the community-acquired MRSA USA 300/USA 400 group (i.e., both pulsed-field type and antimicrobial susceptibility pattern-defined community-acquired MRSA $[n=244]$ ).

¥ Black race compared with white race and other race combined.

§ Analysis restricted to data that were limited to those skin and soft-tissue infections in which a MRSA isolate was available for pulsed-field gel electrophoresis ( $n=159$ community-acquired MRSA infections).

(20). However, standard in vitro susceptibility testing does not differentiate between msr(A)-mediated and MSLBimediated resistance in $S$. aureus. Inducible clindamycin resistance can be detected in the laboratory by performing a "D-zone test." In the test, the clindamycin zone of inhibition is blunted when a clindamycin and erythromycin susceptibility disk are placed side by side on an agar plate containing the $S$. aureus isolate, resulting in a $\mathrm{D}$ shape when inducible clindamycin resistance is present (21). In areas with a high prevalence of community-acquired MRSA infections, laboratories should consider routine implementation of testing for inducible clindamycin resistance.

Incision and drainage without adjunctive antimicrobial agent therapy has often been used as a primary treatment method for skin abscesses with successful outcomes, and this may be applicable for community-acquired MRSA abscesses. Limited data from immunocompetent children suggest that this approach may be successful for abscesses less than $5 \mathrm{~cm}$ in diameter (22). In addition, data from San Francisco have shown that most patients with MRSA skin and soft-tissue infections who received treatment with antibiotics that were not active against MRSA after initial incision and drainage experienced clinical resolution (14). However, further data on treatment outcomes for incision and drainage, with or without adjunctive antimicrobial agent therapy, of community-acquired MRSA skin and soft-tissue infection are needed to determine the efficacy of these methods, as well as to define the risk for recurrence, which may be higher since community-acquired MRSA strains often possess Panton-Valentine leukocidin.

Previous studies have classified patients with MRSA 
infections who were hospitalized within the previous year as having health care-associated MRSA (1). In our study of community-onset $S$. aureus skin and soft-tissue infection, we did not use hospitalization within the previous year as an exclusion criterion when defining community-acquired MRSA. Although previous hospitalization was seen less frequently in patients with community-acquired MRSA than in patients in the other MRSA group or the MSSA group, we observed a history of hospitalization within 1 year in $18 \%$ of patients with a USA 300 pulsed-field type. Thus, a history of hospitalization within 1 year should not be used to exclude the possibility of community-acquired MRSA. Whether acquisition of the community-acquired MRSA USA 300 clone is occurring in the community or in health care settings among those with a history of hospitalization is unknown. Acquisition is probably occurring in both settings on the basis of evidence of community-acquired MRSA transmission in both community settings and, more recently, in health care settings $(2-5,23-27)$.

In our study, black persons were more likely to have skin and soft-tissue infections caused by the communityacquired MRSA USA 300/USA 400 group compared with those with MSSA infections. Other studies have also demonstrated an association between nonwhite race and community-acquired MRSA (1). The reason for this association is unclear, but the association parallels that seen in other invasive bacterial infections, such as those caused by Streptococcus pneumoniae $(28,29)$. Persons in the community-acquired MRSA USA 300/USA 400 group were younger than persons in the other MRSA group, a finding that was also confirmed by previous investigations (1). Younger age may be associated with more frequent close contact between individuals or intrafamilial transmission, child care attendance, less hygienic practices, more frequent skin trauma, and increased rates of incarceration, which have all been implicated in outbreaks of community-acquired MRSA skin and soft-tissue infection $(2-5,30)$. When compared with persons in both the MSSA group and the other MRSA group, persons in the communityacquired MRSA USA 300/USA 400 group were less likely to have been hospitalized in the preceding 12 months. This suggests that spread of the USA 300 clone is primarily occurring in the community, with a smaller portion potentially acquired in a health care setting. Whether this association will remain constant as more patients with community-acquired MRSA infections are hospitalized, which may increase transmission of community-associated strains within hospitals or may even establish these strains as endemic strains within hospitals, will require continued clinical and molecular observation.

Some MRSA isolates were not available for molecular typing by PFGE, and we relied on isolate antimicrobial susceptibility pattern to define community-acquired MRSA in these cases. For these cases, misclassification bias is possible. However, the similarities in demographic characteristics, underlying medical conditions, and other find- ings from our study observed between pulsed-field typedefined and antibiogram-defined community-acquired MRSA suggest that misclassification bias was limited. In addition, we conducted separate analyses using data that were restricted to those patients whose MRSA isolates were available for PFGE. These analyses demonstrated findings similar to those of the overall analysis, which incorporated both pulsed-field type-defined and antibiogram-defined cases. Furthermore, in the event of misclassification, the overall proportion of skin and soft-tissue infections caused by the community-acquired MRSA USA 300/USA 400 group was most likely underestimated since isolates with fluoroquinolone resistance, which has been reported in some community-acquired MRSA isolates, were defined as other MRSA $(1,20)$. We retrospectively reviewed medical records of patients with $S$. aureus skin and soft-tissue infections. Given our study design, confounding of the identified risk factors by unmeasured factors (for example, previous incarceration and day care attendance) or by unavailable data (for example, HIV status) is possible. Furthermore, caution must be used when interpreting the identified associations with community-acquired MRSA, and causal relationships should not be inferred. Future studies using prospective patient interviews are needed to fully define the contribution of these unmeasured risk factors to the risk for developing a skin and soft-tissue infection from community-acquired MRSA.

In an urban Atlanta hospital and its affiliated clinics, the community-acquired MRSA USA 300 clone has become the most common cause of community-onset $S$. aureus skin and soft-tissue infections. Improved recognition by physicians that community-acquired MRSA is the major cause of staphylococcal skin and soft-tissue infection is needed to ensure that appropriate therapy is initiated and to reduce the risk for horizontal transmission in health care settings. Continued hospital-based and population-based surveillance will be required to fully understand the magnitude and ongoing evolution of community-acquired MRSA infections.

From Emory University School of Medicine and Grady Memorial Hospital, Atlanta, Georgia.

Acknowledgments: The authors thank Fred Tenover, Linda McDougal, Sigrid McAllister, and Gregory Foshiem at the Centers for Disease Control and Prevention for performing the Panton-Valentine leukocidin and SCCmec analyses and for providing images containing USA-type strains for comparison. They also thank John Boring for his assistance with calculating prevalence ratios and advice on other statistical considerations.

Grant Support: In part by the Emory Medical Care Foundation, the Emory Mentored Clinical Research Scholars Program (National Institutes of Health/National Center for Research Resources [K12 RR 017643]), and the National Institutes of Health/National Institute of Allergy and Infectious Diseases (K23 AI054371).

Potential Financial Conflicts of Interest: None disclosed. 
Requests for Single Reprints: Henry M. Blumberg, MD, Division of Infectious Diseases, Emory University School of Medicine, 49 Jesse Hill Drive, Atlanta, GA 30303; e-mail, henry.m.blumberg@emory.edu.

Current author addresses and author contributions are available at www .annals.org.

\section{References}

1. Naimi TS, LeDell KH, Como-Sabetti K, Borchardt SM, Boxrud DJ, Etienne J, et al. Comparison of community- and health care-associated methicillinresistant Staphylococcus aureus infection. JAMA. 2003;290:2976-84. [PMID: 14665659]

2. Methicillin-resistant Staphylococcus aureus infections in correctional facilitiesGeorgia, California, and Texas, 2001-2003. MMWR Morb Mortal Wkly Rep. 2003;52:992-6. [PMID: 14561958]

3. Methicillin-resistant Staphylococcus aureus infections among competitive sports participants - Colorado, Indiana, Pennsylvania, and Los Angeles County, 20002003. MMWR Morb Mortal Wkly Rep. 2003;52:793-5. [PMID: 12931079]

4. Outbreaks of community-associated methicillin-resistant Staphylococcus aureus skin infections-Los Angeles County, California, 2002-2003. MMWR Morb Mortal Wkly Rep. 2003;52:88. [PMID: 12588006].

5. Begier EM, Frenette K, Barrett NL, Mshar P, Petit S, Boxrud DJ, et al. A high-morbidity outbreak of methicillin-resistant Staphylococcus aureus among players on a college football team, facilitated by cosmetic body shaving and turf burns. Clin Infect Dis. 2004;39:1446-53. [PMID: 15546080]

6. McDougal LK, Steward CD, Killgore GE, Chaitram JM, McAllister SK, Tenover FC. Pulsed-field gel electrophoresis typing of oxacillin-resistant Staphylococcus aureus isolates from the United States: establishing a national database. J Clin Microbiol. 2003;41:5113-20. [PMID: 14605147]

7. Okuma K, Iwakawa K, Turnidge JD, Grubb WB, Bell JM, O'Brien FG, et al. Dissemination of new methicillin-resistant Staphylococcus aureus clones in the community. J Clin Microbiol. 2002;40:4289-94. [PMID: 12409412]

8. Diep BA, Sensabaugh GF, Somboona NS, Carleton HA, Perdreau-Remington F. Widespread skin and soft-tissue infections due to two methicillin-resistant Staphylococcus aureus strains harboring the genes for Panton-Valentine leucocidin. J Clin Microbiol. 2004;42:2080-4. [PMID: 15131173]

9. Baggett HC, Hennessy TW, Rudolph K, Bruden D, Reasonover A, Parkinson A, et al. Community-onset methicillin-resistant Staphylococcus aureus associated with antibiotic use and the cytotoxin Panton-Valentine leukocidin during a furunculosis outbreak in rural Alaska. J Infect Dis. 2004;189:1565-73. [PMID: 15116291]

10. National Committee for Clinical Laboratory Standards. Methods for Dilution Antimicrobial Susceptibility Tests for Bacteria that Grow Aerobically; Approved Standard. Document M7-A5. vol. 20, no. 2. 5th ed. Wayne, PA: National Committee for Clinical Laboratory Standards; 2000.

11. Bannerman TL, Hancock GA, Tenover FC, Miller JM. Pulsed-field gel electrophoresis as a replacement for bacteriophage typing of Staphylococcus aureus. J Clin Microbiol. 1995;33:551-5. [PMID: 7751356]

12. Tenover FC, Arbeit RD, Goering RV, Mickelsen PA, Murray BE, Persing $\mathrm{DH}$, et al. Interpreting chromosomal DNA restriction patterns produced by pulsed-field gel electrophoresis: criteria for bacterial strain typing. J Clin Microbiol. 1995;33:2233-9. [PMID: 7494007]

13. Lina G, Piémont Y, Godail-Gamot F, Bes M, Peter MO, Gauduchon V, et al. Involvement of Panton-Valentine leukocidin-producing Staphylococcus aureus in primary skin infections and pneumonia. Clin Infect Dis. 1999;29:1128-32. [PMID: 10524952]

14. Young DM, Harris HW, Charlebois ED, Chambers H, Campbell A, Per-
dreau-Remington F, et al. An epidemic of methicillin-resistant Staphylococcus aureus soft tissue infections among medically underserved patients. Arch Surg. 2004;139:947-51; discussion 951-3. [PMID: 15381611]

15. Recommendations for preventing the spread of vancomycin resistance. Recommendations of the Hospital Infection Control Practices Advisory Committee (HICPAC). MMWR Recomm Rep. 1995;44:1-13. [PMID: 7565541]

16. Campaign to Prevent Antimicrobial Resistance in Healthcare Settings: Tools for Clinicians. Atlanta: Centers for Disease Control and Prevention. Accessed at www.cdc.gov/drugresistance/healthcare/patients.htm on 11 January 2006.

17. Marcinak JF, Frank AL. Treatment of community-acquired methicillin-resistant Staphylococcus aureus in children. Curr Opin Infect Dis. 2003;16:265-9. [PMID: 12821819]

18. Lewis JS 2nd, Jorgensen JH. Inducible clindamycin resistance in Staphylococci: should clinicians and microbiologists be concerned? Clin Infect Dis. 2005; 40:280-5. [PMID: 15655748]

19. Frank AL, Marcinak JF, Mangat PD, Tjhio JT, Kelkar S, Schreckenberger PC, et al. Clindamycin treatment of methicillin-resistant Staphylococcus aureus infections in children. Pediatr Infect Dis J. 2002;21:530-4. [PMID: 12182377] 20. Ross JI, Eady EA, Cove JH, Cunliffe WJ, Baumberg S, Wootton JC. Inducible erythromycin resistance in staphylococci is encoded by a member of the ATP-binding transport super-gene family. Mol Microbiol. 1990;4:1207-14. [PMID: 2233255]

21. National Committee for Clinical Laboratory Standards. Performance Standards for Antimicrobial Susceptibility Testing: 14th Informational Supplement. Document M100-S14. Wayne, PA: National Committee for Clinical Laboratory Standards; 2004.

22. Lee MC, Rios AM, Aten MF, Mejias A, Cavuoti D, McCracken GH Jr, et al. Management and outcome of children with skin and soft tissue abscesses caused by community-acquired methicillin-resistant Staphylococcus aureus. Pediatr Infect Dis J. 2004;23:123-7. [PMID: 14872177]

23. Saiman L, O'Keefe M, Graham PL 3rd, Wu F, Saïd-Salim B, Kreiswirth B, et al. Hospital transmission of community-acquired methicillin-resistant Staphylococcus aureus among postpartum women. Clin Infect Dis. 2003;37:1313-9. [PMID: 14583864]

24. Kourbatova EV, Halvosa JS, King MD, Ray SM, White N, Blumberg HM. Emergence of community-associated methicillin-resistant Staphylococcus aureus USA 300 clone as a cause of health care-associated infections among patients with prosthetic joint infections. Am J Infect Control. 2005;33:385-91. [PMID: 16153484]

25. Healy CM, Hulten KG, Palazzi DL, Campbell JR, Baker CJ. Emergence of new strains of methicillin-resistant Staphylococcus aureus in a neonatal intensive care unit. Clin Infect Dis. 2004;39:1460-6. [PMID: 15546082]

26. Charlebois ED, Perdreau-Remington F, Kreiswirth B, Bangsberg DR, Ciccarone D, Diep BA, et al. Origins of community strains of methicillin-resistant Staphylococcus aureus. Clin Infect Dis. 2004;39:47-54. [PMID: 15206052]

27. Seybold U, Kourbatova EV, Johnson JG, Halvosa SJ, Wang YF, King MD, et al. Emergence of community-associated methicillin-resistant Staphylococcus aureus USA300 genotype as a major cause of health care-associated blood stream infections. Clin Infect Dis. 2006;42:647-56. [PMID: 16447110]

28. Pastor P, Medley F, Murphy TV. Invasive pneumococcal disease in Dallas County, Texas: results from population-based surveillance in 1995. Clin Infect Dis. 1998;26:590-5. [PMID: 9524828]

29. Harrison LH, Dwyer DM, Billmann L, Kolczak MS, Schuchat A. Invasive pneumococcal infection in Baltimore, Md: implications for immunization policy. Arch Intern Med. 2000;160:89-94. [PMID: 10632309]

30. Dietrich DW, Auld DB, Mermel LA. Community-acquired methicillinresistant Staphylococcus aureus in southern New England children. Pediatrics. 2004;113:e347-52. [PMID: 15060266] 


\section{Annals of Internal Medicine}

Current Author Addresses: Dr. King: Beacon Clinic, 1136 Alpine Avenue, Suite 205, Boulder, CO 80304.

Drs. Ray and Blumberg and Ms. Humphrey: Division of Infectious Diseases, Emory University School of Medicine, 49 Jesse Hill Drive, Atlanta, GA 30303.

Dr. Wang: Department of Pathology and Laboratory Medicine, Emory University School of Medicine, Grady Memorial Hospital, P.O. Box 26248, 80 Jesse Hill Drive, Atlanta, GA 30303.

Dr. Kourbatova: Division of Tuberculosis and Lung Diseases, Samara State Medical University, Pionerskaya Street, 48, 443099 Samara, Russia.

Author Contributions: Conception and design: M.D. King, H.M. Blumberg.
Analysis and interpretation of the data: M.D. King, B.J. Humphrey, Y.F. Wang, E.V. Kourbatova, S.M. Ray, H.M. Blumberg.

Drafting of the article: M.D. King, S.M. Ray, H.M. Blumberg.

Critical revision of the article for important intellectual content: M.D. King, B.J. Humphrey, Y.F. Wang, E.V. Kourbatova, S.M. Ray, H.M. Blumberg.

Final approval of the article: M.D. King, B.J. Humphrey, Y.F. Wang, E.V. Kourbatova, S.M. Ray, H.M. Blumberg.

Statistical expertise: M.D. King, E.V. Kourbatova.

Obtaining of funding: M.D. King, H.M. Blumberg.

Administrative, technical, or logistic support: M.D. King, H.M. Blumberg.

Collection and assembly of data: M.D. King, B.J. Humphrey. 